
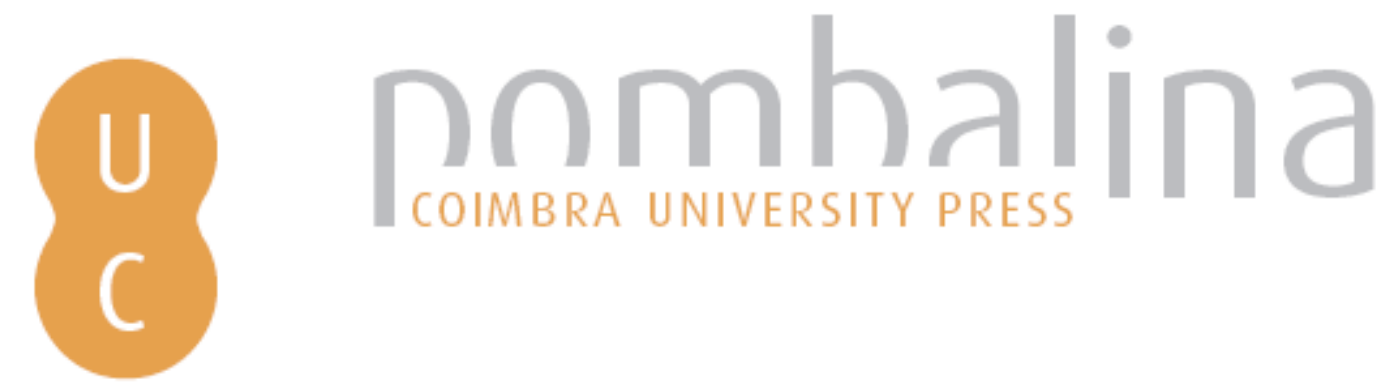

\title{
La formación en biblioteconomía y documentación con compromiso social: la educación sobre Derechos Humanos en las titulaciones de España y Portugal
}

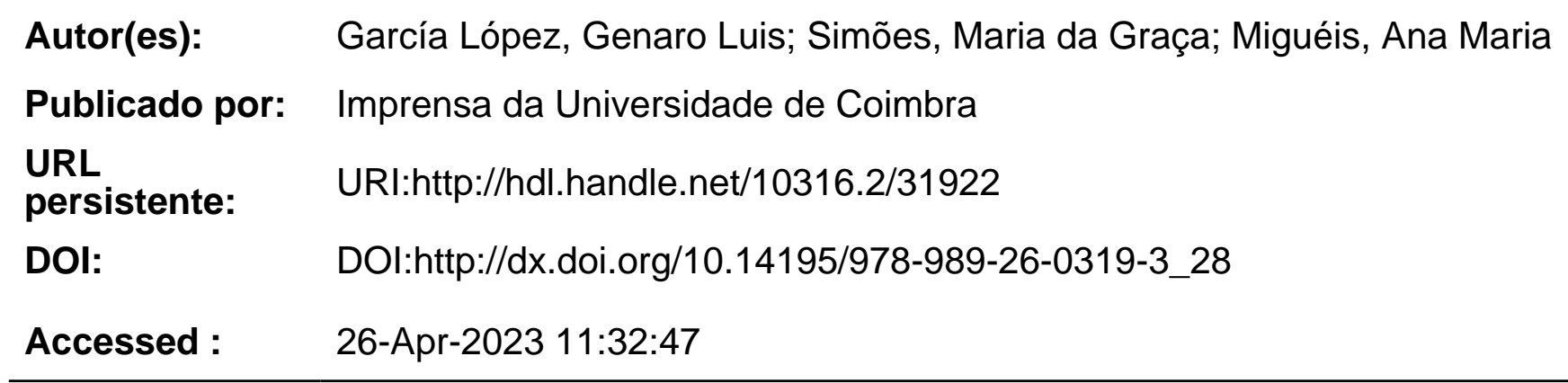

A navegação consulta e descarregamento dos títulos inseridos nas Bibliotecas Digitais UC Digitalis, UC Pombalina e UC Impactum, pressupõem a aceitação plena e sem reservas dos Termos e Condições de Uso destas Bibliotecas Digitais, disponíveis em https://digitalis.uc.pt/pt-pt/termos.

Conforme exposto nos referidos Termos e Condições de Uso, o descarregamento de títulos de acesso restrito requer uma licença válida de autorização devendo o utilizador aceder ao(s) documento(s) a partir de um endereço de IP da instituição detentora da supramencionada licença.

Ao utilizador é apenas permitido o descarregamento para uso pessoal, pelo que o emprego do(s) título(s) descarregado(s) para outro fim, designadamente comercial, carece de autorização do respetivo autor ou editor da obra.

Na medida em que todas as obras da UC Digitalis se encontram protegidas pelo Código do Direito de Autor e Direitos Conexos e demais legislação aplicável, toda a cópia, parcial ou total, deste documento, nos casos em que é legalmente admitida, deverá conter ou fazer-se acompanhar por este aviso.

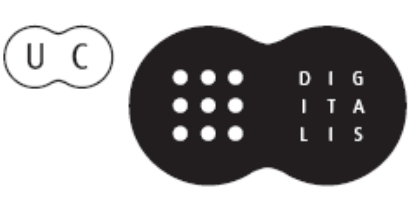


Maria Manuel Borges

Elias Sanz Casado

Coordenação

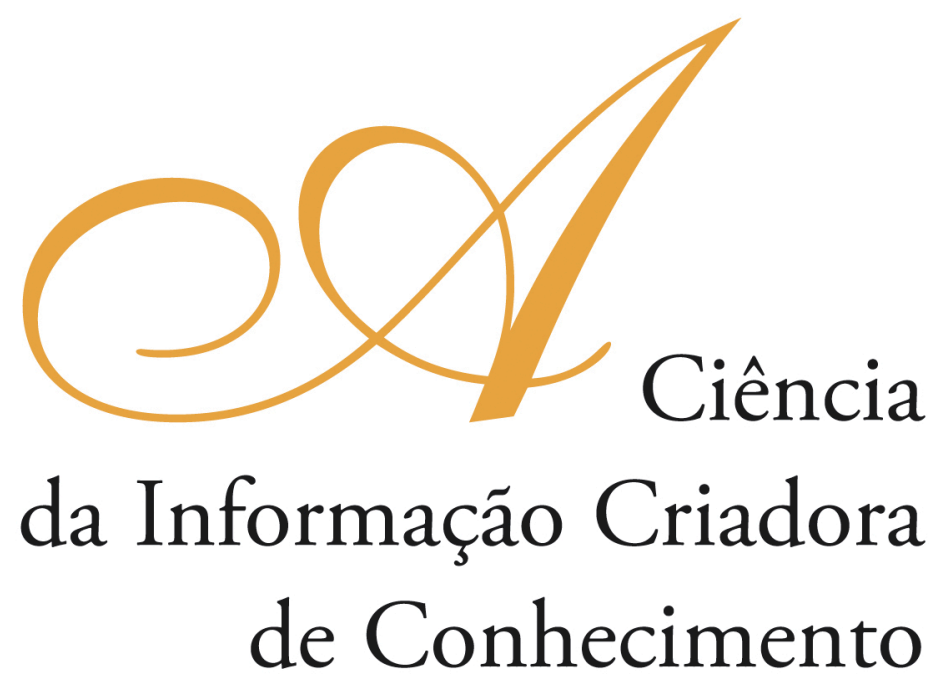

Vol. I

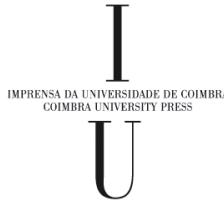

- COIMBRA 2009 
La formación en Biblioteconomía y Documentación

CON COMPRomiso SOCIAL: La EDUCACIÓn SOBRE DERECHOS

Humanos en las titulaciones de España y Portugal

\author{
Genaro Luis García López \\ Universidade de Salamanca (Espanha) \\ Maria da Graça Simóes \\ Universidade de Coimbra (Portugal)
}

Ana Maria Miguéis

Universidade de Coimbra (Portugal)

\title{
Resumen
}

Se analiza cuál es la situación de los Derechos Fundamentales en el mundo, se comentan los planes de acción de Naciones Unidas para la educación en Derechos Humanos y se enfatiza en la necesidad de incluir contenidos, competencias y destrezas sobre estas cuestiones en los currícula de los distintos niveles educativos. A continuación, se han estudiado los planes de estudio de los grados en Información y Documentación en Espańa y Portugal, para analizar el nivel de cumplimiento con las pautas establecidas por la ONU y se realizan propuestas para futuras modificaciones con el fin de que se puedan adecuar a las recomendaciones internacionales sobre este asunto.

\section{Abstract}

The notion of education as fundamental Human Right, which is considered to be essential for the exercise of other human rights by United Nations and UNESCO, are discussed. The need to introduce contents and skills about this matter at all educational level, especially Library and Documentation graduation studies in Spain and Portugal are analysed in comparison to United Nations recommendations. An outline of proposals to change current situation and implement curriculum for these graduation studies in line with international recommendations.

\section{Introducción}

Dos profesores de la Universidad Complutense de Madrid, Pedro López López e Inmaculada Vellosillo (2008: 13), comienzan un libro reciente refiriendo como poco después del 11-S en una universidad brasileńa, bajo una foto de Osama Bin Laden aparecía el letrero «Bin Laden. Ingeniero. Formar un profesional es fácil; lo difícil es formar un ciudadano».

Leer información de ese tipo seguramente nos puede llamar la atención, pero también nos hará reflexionar sobre la finalidad última de todos esos ańos que niños, adolescentes y adultos jóvenes (o no tan jóvenes) pasan en las aulas a lo largo de sus vidas. 
En este artículo ${ }^{1}$ se expone cómo la Educación en Derechos Humanos tiene que estar presente en las aulas en todos los niveles educativos, mostrando algunas experiencias y poniendo de manifiesto que ése es el camino para conseguir un mundo mejor. Si queremos que el eslogan que afirma que «Otro mundo (mejor) es posible» se haga realidad, tendremos que comenzar por inculcar a nuestros hijos la esperanza de una vida mejor marcada por el respeto, la tolerancia y los valores de ciudadanía, el compromiso y la defensa de los valores universales.

\section{La educación en valores y derechos universales en la sociedad actual}

El Plan de acción del programa mundial para la educación en Derechos Humanos de la ONU (Naciones Unidas, 2006) vigente hasta este año señala que la educación en Derechos Humanos debe proporcionar conocimientos sobre los DD.HH. y los mecanismos para protegerlos, y además transmitir las aptitudes necesarias para promover, defender y aplicar los Derechos Fundamentales en la vida cotidiana; debiendo además transmitir los principios fundamentales de los Derechos Humanos, consolidando su interdependencia, indivisibilidad y universalidad.

Siguiendo a Naciones Unidas (2006) podemos afirmar que el enfoque que se le ha de dar a la educación debe tener su base en los Derechos Fundamentales y « [...] la educación en derechos humanos forma parte del derecho del niño a recibir una educación de alta calidad, en que no sólo se enseñe lectura, escritura o aritmética, sino que además se fortalezca la capacidad del niño de disfrutar todos los derechos humanos y se fomente una cultura en que prevalezcan los valores de los derechos humanos».

Para conseguir ese gran objetivo Naciones Unidas identifica cinco grandes componentes que incidirán en su consecución:

a) Políticas educativas: compromisos de los gobiernos, leyes, planes de acción, planes de estudio... que deben elaborarse de forma participativa y con el enfoque de los Derechos Humanos.

b) Aplicación de políticas: seguir una estrategia coherente de aplicación, con suficientes recursos, rendición de cuentas, implicación de distintos agentes...

c) Entorno de aprendizaje: desarrollo cognitivo, pero también desarrollo social y emocional, en un ambiente de comprensión, respeto y responsabilidad mutuos.

d) Enseńanza y aprendizaje: enfoque holístico de la enseñanza y el aprendizaje, integrando los conceptos y prácticas de los Derechos Básicos lo antes posible en todos los aspectos de la educación.

e) Formación y perfeccionamiento profesional del personal docente: fomentar el conocimiento de los Derechos Esenciales por parte del profesorado con el fin de que puedan transmitir sus valores.

${ }^{1}$ Esta comunicación es una adaptación, para aplicarla al campo de las titulaciones en Información y Documentación, de la ponencia presentada al II Congreso Internacional de Derecho Educativo, celebrado en Tuxtla Gutiérrez, Chiapas (México) en marzo de 2009 titulada: La formación en Derechos Humanos: hacia una educación con sentido. 
En ese contexto se considera la educación en DD.HH. como una prioridad nacional de cualquier país, que quiera solucionar los problemas de los sistemas educativos actuales, así como aumentar la eficacia de su sistema nacional de enseñanza con el fin de que sirva para el desarrollo económico, social y político.

Un "amante" de los valores que venimos comentando, como es el chileno Roberto Garretón (2006) nos recuerda a Paulo Freire para seńalar la importancia de la educación en Derechos Humanos, así como la necesidad de acometer desde un punto de vista político la defensa de los Derechos Fundamentales, comenzando desde la educación:

Tiene razón, como siempre, Paulo Freire, al afirmar que no existe "educación neutral". Busca objetivos políticos, porque la justicia es tanto conmutativa (responsabilidad, no a la impunidad, nunca más) como distributiva (derechos económicos y sociales). Pero al hablar del sentido político de la educación, y justamente, la educación en derechos humanos, debemos aceptar otra regla, que el mismo Paulo Freire describe así: "iqué insensatez es pretender educar para los derechos humanos encerrados en un aula algunas horas a la semana!". La educación en derechos humanos desde luego comprende los códigos formales de los derechos humanos: declaraciones, tratados, sentencias, etc., pero ello no basta, sino que se requiere una educación transversal, a través de todas las cátedras, que es lo que los especialistas llaman el "currículo oculto”, que más allá de conocimientos, van formando cultura.

\section{La formación de ciudadanos responsables y conocedores de sus derechos en los distintos niveles educativos}

Si concebimos la ciudadanía «como participación en la vida social y política, una participación que, si falta, vacía de contenido la democracia» (López López, Vellosillo González, 2008 : 13) podemos comprobar también la necesidad de que en las aulas se impartan contenidos sobre democracia, participación política y Derechos Humanos; pues no se trata sólo de transmitir determinados conocimientos, sino también ciertos valores, actitudes, así como formas de estar y de entender el mundo.

Si queremos una democracia plenamente desarrollada, necesitamos también ciudadanos: formados, informados, que conozcan los asuntos públicos y que desarrollen una actividad proactiva por el bien común. Y para ello se necesita tanto derechos reconocidos como "una ciudadanía proactiva» (Giner, 2004 : 146).

López López y Vellosillo González (2008 : 14) proponen cuatro campos en los que actuar para avanzar hacia una ciudadanía proactiva:

- El reforzamiento de la esfera pública.

- La potenciación de vías de participación ciudadana.

- La independencia de los medios de comunicación.

- El sistema educativo.

El dilema sobre si hay que formar o no a los ciudadanos parece residir fundamentalmente en la importancia que se le quiera dar a la esfera pública y a las políticas (en este caso educativas y de información). Así, siguiendo a Mattelart (2002), 
podríamos concluir que en la actual sociedad neoliberal el centro de gravedad se traslada de la esfera de la ciudadanía a la del consumo.

En este sentido, según Jares (2005), la concepción neoliberal de la educación supone que:

- La educación pasa de ser un derecho a un bien de consumo.

- Los centros educativos se conviertan en empresas.

- El mercado y la libre competencia entre centros se consideran como garantes de calidad y libertad.

- Se vuelva a una supuesta neutralidad y objetividad.

- Se refuerce la concepción utilitarista de la calidad y la eficacia.

- Se fomente el individualismo y el conformismo.

Para contrarrestar todos esos efectos negativos es necesaria una educación en valores democráticos y de Derechos Fundamentales. En realidad la participación activa comporta diversos beneficios (Escámez Sánchez, 2003 : 197-198):

- La elección compartida de las prioridades sociales: posibilidad de discutir, debatir y seleccionar los valores que rigen las prioridades de la sociedad.

- El ejercicio de la autonomía responsable: avanzar en un comportamiento a la altura de la dignidad humana, al convertir a cada persona en actor de su propia vida y de la de su comunidad.

- La creación de capital social: donde es más fácil resolver los dilemas de la acción colectiva.

- El desarrollo económico de los pueblos: se reducen las hambrunas y los dirigentes se ven obligados a atender las necesidades económicas de los ciudadanos (según indica el premio Nobel de economía Amartya Sen).

\section{Bases normativas del derecho internacional sobre la educación en Derechos Fundamentales}

El artículo 26 de la Declaración Universal de los Derechos Humanos establece que:

1. Toda persona tiene derecho a la educación. La Educación debe ser gratuita, al menos en lo concerniente a la instrucción elemental y fundamental. La instrucción elemental será obligatoria. La instrucción técnica y profesional habrá de ser generalizada; el acceso a los estudios superiores será igual para todos, en función de los méritos respectivos.

2. La educación tendrá por objeto el pleno desarrollo de la personalidad humana y el fortalecimiento del respeto a los derechos humanos y a las libertades fundamentales; favorecerá la comprensión, la tolerancia y la amistad entre todas las naciones y todos los grupos étnicos o religiosos; y promoverá el desarrollo de las actividades de las Naciones Unidas para el mantenimiento de la paz.

Esto ha servido de base para diversos acuerdos internacionales que han considerado la educación en Derechos Humanos como pilar fundamental de la educación para promover la tolerancia y el respeto. Así, diversos instrumentos internacionales (que han desarrollado la Declaración Universal) han incorporado disposiciones relativas a la educación en DD.HH. 
Entre éstos, podemos destacar:

- El Pacto Internacional de Derechos Económicos, Sociales y Culturales (1966): artículo 13.1 .

- La Declaración de los Derechos del Niño (1959): principio 7.

- La Convención sobre los Derechos del Niño (1989): artículo 29.

- La Convención sobre la eliminación de todas las formas de discriminación contra la mujer (1979): artículo 10.

- La Convención Internacional sobre la Eliminación de todas las formas de Discriminación Racial (1965): artículo 7.

- La Convención de la UNESCO relativa a la lucha contra la discriminación en la esfera de la enseñanza (1960): artículo 5.1.a.

- El Convenio 169 de la OIT sobre pueblos indígenas y tribales en países independientes (1989): artículos 26 y 28.

- La Declaración y Programa de Acción de Viena (1994): parte I.

- La Carta de la Organización de Estados Americanos (1948): artículo 3.

Así, por ejemplo, la citada Declaración y Programa de Acción de Viena de 1994 seńala en su apartado "D. Educación en materia de derechos humanos»:

78. La Conferencia Mundial de Derechos Humanos considera que al educación, la capacitación y la información pública en materia de derechos humanos son indispensables para establecer y promover relaciones estables y armoniosas entre las comunidades y para fomentar la comprensión mutua, la tolerancia y la paz.

79. Los Estados deben tratar de eliminar el analfabetismo y deben orientar la educación hacia el pleno desarrollo de la personalidad humana y el fortalecimiento del respeto de los derechos humanos y las libertades fundamentales. La Conferencia Mundial de Derechos Humanos pide a todos los Estados e instituciones que incluyan los derechos humanos, el derecho humanitario, la democracia y el imperio de la ley como temas de los programas de estudio de todas las instituciones de enseñanza académica y no académica.

80. La educación en materia de derechos humanos debe abarcar la paz, la democracia, el desarrollo y la justicia social, tal como se dispone en los instrumentos internacionales y regionales de derechos humanos, a fin de lograr la comprensión y sensibilización de todos acerca de los derechos humanos con objeto de afianzar la voluntad de lograr su aplicación a nivel universal.

\section{Pautas y programas de Naciones Unidas sobre educación en Derechos Humanos}

Ya en la Conferencia Mundial sobre Derechos Humanos, que se celebró en Viena en junio de 1993, se concluyó solicitar a los Estados e instituciones «la introducción de los DD.HH., el derecho internacional humanitario, la democracia y el imperio de la ley como temas de los programas de estudio de todas las instituciones de enseñanza académica y no académica».

Dos años después, arrancó el Decenio de las Naciones Unidas para la educación en la esfera de los Derechos Humanos 1995-2004 (Naciones Unidas: 1996) que definía la educación en la esfera de los DD.HH. como: «[...] el conjunto de actividades de 
capacitación, difusión e información encaminadas a crear una cultura universal en la esfera de los derechos humanos, actividades que se realizan transmitiendo conocimientos y moldeando actitudes».

El citado documento establecía los siguientes fines:

a) Fortalecer el respeto de los derechos humanos y las libertades fundamentales.

b) Desarrollar plenamente la personalidad humana y el sentido de la dignidad del ser humano.

c) Promover la comprensión, la tolerancia, la igualdad entre los sexos y la amistad entre todas las naciones, las poblaciones indígenas y los grupos raciales, nacionales, étnicos, religiosos y lingüísticos.

d) Facilitar la participación eficaz de todas las personas en una sociedad libre.

e) Intensificar las actividades de las Naciones Unidas en la esfera del mantenimiento de la paz.

Ese mismo documento insistía en la concepción de los Derechos Humanos como:

- Indivisibles.

- Herramienta de inclusión de la mujer en pie de igualdad con el hombre.

- Formación fundamental, tanto en la educación formal, como en la educación profesional, así como en la no reglada.

- Conocimientos importantes en la vida cotidiana de la gente, para que no sean sólo normas abstractas, sino que se integren en la realidad de las circunstancias sociales, económicas, culturales y políticas de las comunidades.

- Elemento para propiciar la participación democrática y para promover el progreso económico y social, así como el desarrollo sostenible.

- Medio para luchar contra prejuicios por razones de sexo, raza o de otro tipo.

- Medio para inculcar aptitudes y conocimientos, influyendo en las actitudes y conducta de los educandos.

Como se puede comprobar una formación en valores de respeto, compromiso y tolerancia que deberían ser objetivos comunes de cualquier norma sobre educación. Además, consideramos muy acertada la postura de Garretón (2006) que señala que todo programa de educación de Derechos Humanos deberá tener como hilos conductores los principales valores que emanan de la Declaración Universal de los DD.HH. y los textos declarativos y convencionales ulteriores:

- El principio de la dignidad de todos los miembros de la familia humana: que es intrínseco e inderogable en toda persona.

- El principio de no discriminación: exigencia de igualdad de todos los seres humanos. Desarrollado específicamente, en materia educativa, en una Convención de la UNESCO, adoptada en 1960.

- El principio de democracia: punto de partida para garantizar el respeto a los Derechos Fundamentales.

En el mismo sentido otras organizaciones internacionales han incidido en la necesidad de contar con contenidos de Derechos Humanos en los currícula universitarios. Así, la Sección Española de Amnistía Internacional (2008 y 2008a) ha pedido a las autoridades espańolas: 
- Que se incluyan contenidos obligatorios de DD.HH. en los planes de estudio de todos los niveles educativos.

- Que se promueva el incremento de las horas lectivas obligatorias de estos contenidos, para asimilarnos a otros países europeos.

- Que se incluya la educación en DD.HH. en la formación y capacitación profesional de los docentes, garantizando también la formación permanente del profesorado responsable de impartir la asignatura de Educación para la Ciudadanía y los Derechos Humanos.

- Que se introduzcan contenidos de DD.HH. en los criterios de verificación de los nuevos planes de estudio que deben aplicar las agencias externas de evaluación.

- Que las autoridades universitarias incorporen el conocimiento en DD.HH. dentro de la relación de destrezas, capacidades y competencias generales que cualquier estudiante debe adquirir durante su formación universitaria.

- Que siguiendo las recomendaciones internacionales, los materiales didácticos sean revisados por expertos en educación en Derechos Humanos (en el caso de la enseñanza primaria y secundaria).

- Que el Gobierno cree una Comisión Nacional de Seguimiento de la Educación en Derechos Humanos.

- Que la adaptación de las leyes educativas garantice el cumplimento de las metas y objetivos establecidos por el Programa Mundial de Educación en Derechos Humanos.

\section{La impartición de Derechos Humanos en los grados de Información y Documentación en universidades de España y Portugal}

En lo concerniente a nuestro objeto de estudio, es importante resaltar, entre otros textos legislativos, tanto nacionales como internacionales, la Recomendación relativa a la Convención Europea de los Derechos del Hombre sobre la Educación Universitaria y la Formación Profesional emitida por el Comité de Ministros del Consejo de Europa $(2004)^{2}$, que se aplica naturalmente a Portugal y España.

El motivo de su interés se desprende del hecho de que subraya, en particular, la importancia de la educación universitaria y de los programas de formación profesional como medio de sensibilizar, aplicar, dar cumplimiento y garantizar en la práctica sus principios. Entre otros propósitos intenta que los Estados miembros:

- Aseguren una educación universitaria y una formación profesional, a nivel nacional, adecuados a la referida Convención y jurisprudencia del Tribunal Europeo de los Derechos Humanos.

- Incluyan estas materias como troncales en los planes de estudio de los cursos de derecho, así como en los cursos de ciencias políticas y administrativas.

- Que los DD.HH. sean objeto de estudio de los programas de preparación para los exámenes nacionales de acceso a diversas profesiones jurídicas, así como de la formación inicial y continua destinada a los jueces, fiscales y abogados.

\footnotetext{
${ }^{2}$ http://www.gddc.pt/direitos-umanos/Recomendacoes/RECOMENDA\%C7\%C3O\%20REC\%20(2 004)\%204\%20do\%20Comit\%E9\%20de\%20Ministros.doc
} 
- Proporcionen formación profesional inicial y continua al personal de otros sectores responsables de la aplicación de las leyes y al personal que se encuentra en contacto permanente y directo con personas privadas de libertad, tales como: policía, miembros de las fuerzas de seguridad, personal penitenciario y de hospitales. Se añade a esto el personal de de los servicios de inmigración, como por ejemplo: los servicios de extranjería y fronteras.

Además, con su aplicación se pretende también:

- El refuerzo y la eficacia en la educación universitaria y en la formación profesional.

- El apoyo a iniciativas cuyo objetivo sea formar docentes y formadores especializados en esta área.

- Fomentar iniciativas no estatales que promuevan la sensibilización y el conocimiento del sistema de la Convención, como la implementación de estructuras especializadas en la enseńanza y en la investigación en materia de Derechos Humanos o campañas de sensibilización.

Con base en lo anteriormente expuesto, y en particular en esta Convención, la educación superior en Portugal y España ha debido venir paulatinamente a integrar y a desarrollar, en sus planes curriculares, materias relacionadas con los Derechos Humanos y las actividades con ellos relacionadas.

Testimonio de esta preocupación curricular es lo que se viene observando, especialmente en la década de los ańos noventa e inicios del siglo XXI, sobre todo con la adecuación de los contenidos curriculares al marco del Espacio Europeo de Educación Superior.

En este contexto, apuntamos, para el caso de Portugal, la creación del Centro de Derechos Humanos (CDH) del Ius Gentium Conimbrigae, en el año 2000, en la Faculdade de Direito da Universidade de Coimbra. Este es el primer centro universitario de enseñanza e investigación en el área de los Derechos Humanos en Portugal, pudiéndose destacar entre sus objetivos principales los siguientes:

- La realización de actividades de investigación en esta área.

- La enseñanza y formación a través de la realización de conferencias, seminarios, cursos de verano...

- La realización del curso de posgraduación en Derechos Humanos y Democratización, cuyo propósito se centra en la formación especializada en esta temática de los Derechos Humanos.

- La divulgación y promoción de acciones junto a las entidades que lo soliciten.

En lo relativo a nuestro objeto de análisis, que se circunscribe a las Ciencias de la Información Documental, en general en los grados y posgrados, los planes de estudio están mayoritariamente exentos de estas materias; situación que nos parece preocupante, dada la importancia que las mismas tienen en la formación intelectual y personal de cualquier estudiante.

Tras la revisión de los planes de estudio de los grados integrados en los currícula de acuerdo con las orientaciones del EEES y que se encuentran en vigor desde el curso lectivo 2008-2009, podemos verificar que en ninguno de los dos países existen 
disciplinas específicas sobre Derechos Humanos, observándose no obstante disciplinas relacionadas con ellos como son las de Ética y Deontología.

En concreto, registramos en España, el caso de la Universidad de Salamanca ${ }^{3}$, donde se enseña, en este grado, la asignatura de Ética de la información y la documentación, y de la Universidad Carlos III de Madrid ${ }^{4}$, donde se imparte la asignatura de Ética y deontologia profesional.

Basándonos en idénticos criterios de análisis observamos la misma situación en las universidades portuguesas. Disciplinas sobre Derechos Humanos propiamente dichos tampoco existen. Así todo, se observa, como en el caso de las universidades españolas, la introducción de asignaturas relacionadas con los mismos, siendo el caso de la Universidade Católica Portuguesa y de la Universidade de Evora, las cuales integran en sus planes de estudio, las disciplinas de Direito e Ética ${ }^{5}$ da Informação y de Ética e Deontologia ${ }^{6}$, respectivamente.

En relación también con Portugal, nos parece especialmente relevante la ausencia de materias sobre Derechos Humanos en los planes de estudio de las Universidades de Coimbra y Porto. Su ausencia es más evidente por el hecho de ser referencia en el mundo académico y científico en el área de las Ciencias de la Información, producto de su larga experiencia. Además, se trata de universidades públicas y por lo tanto tienen, desde nuestro punto de vista, una mayor responsabilidad en la sensibilización y divulgación de todos los aspectos relacionados con los Derechos Humanos, al erigirse en garantes del respeto y dignidad del ser humano. Así, si estas universidades comtemplaran estas materias en sus planes de estudio serían un modelo a seguir para otros instituciones académicas similares.

Como docentes y ciudadanos conscientes del interés de estas materias no podemos dejar de manifestar con especial satisfacción el hecho de que la Universidad Complutense, en su plan de estudios de Grado de Información y Documentación (2009-2010), ya incluye una asignatura específica ${ }^{7}$ : Derechos Humanos, Ciudadania y Sociedad de la Información. Algo similar se registra en Portugal, en el caso de la Universidade Portucalense la cual introduce en los planes de estudio ${ }^{8}$ de la Licenciatura en Ciências da Informação e Documentação una disciplina relacionada con los Derechos Humanos: Direito e aspectos éticos da informação.

Por ello, considerando estas disciplinas como muy próximas a las propósitos de los Derechos Humanos, entendemos que los planes de estudio no se encuentran adecuados a los objetivos y competencias que se pretenden inculcar en quien estudia y que en el futuro ejercerá en esta área profesional. Por ello pensamos que deberían existir más disciplinas orientadas, de forma concreta y específica, a los DD.HH., y otras relacionadas con ellos, con el fin de responder cabalmente a las complejidades emergentes de la globalización.

\footnotetext{
${ }^{3}$ http://www.usal.es/webusal/files/plan_estudios_grado_Informacion_documentacion.pdf.

${ }^{4}$ http://www.uc3m.es/portal/page/portal/titulaciones_grado/info_doc/plan.

${ }^{5}$ http://www.facfil.ucp.pt/1ciclo/cid/1ciclo.html\#PLANO\%20CURRICULAR

${ }^{6}$ http://www.uevora.pt/ensinos/cursos/11_ciclo/curso/plano_de_estudos/(codigo)/148

${ }^{7}$ http://www.ucm.es/pags.php?tp=Grados \&a=estudios $\& \mathrm{~d}=$ muestragrado3.php\&idgr=23

${ }^{8}$ http://wwwa.uportu.pt/siaa/Destaques/Dr_Ciencia_Informacao.pdf
} 


\section{Conclusiones: Propuestas para la reforma, y elaboración, de planes de estudio en Información y Documentación}

Consideramos que los problemas actuales que tiene que afrontar el mundo (el hambre, la mala distribución de la riqueza, la crisis económica, la crisis de valores, la falta de concienciación sobre el deterioro del medio ambiente...) necesitan de un compromiso conjunto de toda la sociedad y eso sólo se podrá lograr si desde la escuela (y continuando por todos los niveles educativos) comenzamos a formar a las futuras generaciones en los valores de respeto, compromiso y tolerancia. Lo cual se plasma en el conocimiento de nuestros más elementales derechos, que todos tenemos reconocidos simplemente por haber nacido como personas.

Parafraseando al chileno Roberto Garretón (2006) podemos acabar afirmando que:

La influencia de los derechos humanos -y su instrumento matriz, la Declaración Universal- se ha extendido a todos los ámbitos de la cultura: la filosofía, la ética, la política, la historia, las artes, el derecho, las relaciones internacionales, la economía, la sociología, la antropología, las comunicaciones, la arquitectura, la medicina, la educación, etc. hoy no pueden evitar el tratamiento del tema. En lo jurídico, hoy los derechos humanos ejercen -o debieran ejercer- una influencia cada vez mayor en los derechos constitucional, penal, procesal, civil, laboral, administrativo, penitenciario, policial, militar, ambiental, médico legal, internacional, la filosofía del derecho, sin perjuicio de constituir una nueva y autónoma rama del derecho.

Colocando a los Derechos Humanos en el centro de nuestra vida, en el centro de nuestra actividad educativa, algún día podremos afirmar que la educación, además de formar profesionales, sirve también (y sobre todo) para formar buenas personas. Un reto que tenemos todos por delante y que está en nuestras manos asumir.

Si no queremos, parafraseando al Director de la Sección Española de Amnistía Internacional (Beltrán, 2009) que nuestra sociedad esté regida por "derechos torcidos» será necesaria una implicación colectiva por la defensa de los valores comunes.

Con vistas a la previsible modificación de los planes de estudio ya implantados, así como a la creación de nuevos planes de estudio, se propone incluir distintas competencias, criterios para validación de propuestas de planes de estudio, inclusión de asignaturas específicas, etcétera. Muchas de estas propuestas han sido extraídas de los distintos documentos elaborados por la organización de defensa de los Derechos Humanos Amnistía Internacional (2007, 2008, 2008a, 2008b), ya señaladas en el apartado 5 .

Será recomendable mencionar la necesidad de que los alumnos adquieran valores y competencias entre los que estarán:

- Avanzar en el respeto y promoción de los Derechos Humanos.

- Promover la interdependencia, la indivisibilidad y la universalidad de los Derechos Humanos, incluso los derechos civiles, políticos, económicos, sociales, culturales y medioambientales, así como el derecho al desarrollo.

- Fomentar el respeto y la valoración de las diferencias, así como la oposición a la discriminación por motivos de raza, sexo, orientación sexual, identidad sexual, idioma, 
religión, opinión política o de otra índole, origen nacional, étnico o social, condición física o mental, o por otros motivos.

- Alentar el análisis de problemas críticos e incipientes en materia de Derechos Humanos, en particular la pobreza, los conflictos violentos y la discriminación, para encontrar soluciones compatibles con las normas relativas a los Derechos Humanos.

- Dotar a las comunidades y a las personas de los medios necesarios para determinar sus necesidades en materia de Derechos Humanos y velar por su satisfacción.

- Inspirarse en los principios de Derechos Humanos consagrados en los distintos contextos culturales, y tener en cuenta los acontecimientos históricos y sociales de cada país.

- Fomentar los conocimientos sobre instrumentos y mecanismos para la protección de los Derechos Humanos y la capacidad de aplicarlos a nivel mundial, local, nacional y regional.

- Utilizar métodos pedagógicos participativos que incluyan conocimientos, análisis críticos y técnicas para promover los Derechos Humanos.

- Fomentar entornos de aprendizaje y enseñanza sin temores ni carencias, que estimulen la participación, el goce de los Derechos Humanos y el desarrollo pleno de la personalidad humana.

- Ser pertinentes en la vida cotidiana de los educandos, haciendo que éstos participen en un diálogo sobre los medios de transformar los Derechos Humanos de la expresión de normas abstractas a la realidad de sus condiciones sociales, económicas, culturales y políticas.

Igualmente será necesario elaborar criterios que sirvan para la validación de planes y programas de estudios, entre los cuales deben estar: Incluir competencias y contenidos de Derechos Humanos, defensa de la cultura de la paz, acciones contra la discriminación de distintos tipos, fomento de una cultura cívica, promoción del desarrollo sostenible y protección del medio ambiente.

Finalmente, y no menos importante, es necesario:

- Que se impartan asignaturas obligatorias sobre Derechos Humanos en los grados en Información y Documentación.

- Que se regule el número mínimo de horas lectivas obligatorias de estos contenidos.

- Que se establezca la creación de una Comisión Nacional de Seguimiento de la Educación en Derechos Humanos.

\section{Referências bibliográficas}

AMNISTÍA Internacional. Sección Española (2007), Educación para la Ciudadanía y los Derechos Humanos: declaración: 10 de septiembre de 2007. Accesible en: https://doc.es.amnesty.org/cgibin/ai/BRSCGI?CMD=VERPAG\&PAG=formulario. Fecha de consulta: 3-3-2009.

AMNISTÍA Internacional. Sección Española (2008), Las universidades españolas, a la cola de Europa en formación obligatoria en derechos humanos. Accesible en: https://doc.es.amnesty.org/ cgi-bin/ai/BRSCGI?CMD=VERPAG\&PAG=formulario. Fecha de consulta: 4-3-2009. 
AMNISTÍA Internacional. Sección Española (2008a), Un paso correcto, un desarrollo insuficiente. Valoración por parte de Amnistía Internacional de la asignatura de Educación para la Ciudadania y los Derechos Humanos en España. 2 de octubre de 2008. Accesible en: https:// doc.es.amnesty.org/cgi-bin/ai/BRSCGI?CMD=VERPAG\&PAG=formulario. Fecha de consulta: 8-3-2009.

AMNISTÍA Internacional. Sección Española (2008b), 60 aniversario de la Declaración Universal de los Derechos Humanos: tendencias en los Derechos Humanos durante los últimos 60 años. Accesible en: https://doc.es.amnesty.org/cgi-bin/ai/BRSCGI?CMD=VERPAG\&PAG=form ulario. Fecha de consulta: 8-3-2009.

BELTRÁN, Esteban (2009), Derechos torcidos: tópicos, medias verdades y mentiras sobre pobreza, politica y derechos humanos, Barcelona, Debate.

CONSELHO da Europa. Comité de Ministros (2004), Recomendação(REC)(2004) 4 do Comité de Ministros dirigida aos Estados membros relativa à Convenção Europeia dos Direitos do Homem sobre o Ensino Universitário e a Formação Profissional. Accesible en: http://www. gddc.pt/direitos-humanos/Recomendacoes/RECOMENDA\%C7\%C3O\%20REC\%20(200 4)\%204\%20do\%20Comit\%E9\%20de\%20Ministros.doc. Fecha de consulta: 8-3-2009.

ESCÁMEZ SÁNCHEZ, Juan (2003), «La educación para la participación en la sociedad civil», Revista de educación, número extraordinario Ciudadanía y educación, p. 191-211.

GARRETÓN, Roberto (2006), «El derecho humano a la educación en derechos humanos», en: Seminario internacional Educación y Derechos Humanos. Accesible en: www.ombudsman. cl/pdf/jujuy.pdf. Fecha de consulta: 8-3-2009.

GINER, Salvador (2004), "Ciudadanía, ciudad y mundialización de la sociedad civil», en: A. Montesino, M. Roscales (eds.), Conciencias de la mirada urbana: ciudad, ciudadanía y virtudes civicas, Santander, Límite, p. 145-170.

LÓPEZ LÓPEZ, Pedro; VELLOSILLO GONZÁLEZ, Inmaculada (2008), Educación para la ciudadanía y biblioteca escolar, Gijón (España), Trea.

MATTELART, Armand (2002), «Premisas y contenidos ideológicos de la Sociedad de la Información», en: J. Vidal Beneyto, La ventana global, Madrid, Taurus, p. 65-80.

NACIONES UNIDAS. Asamblea General (1996), A/51/506/Add.1. Informe del alto comisionado de las Naciones Unidas para los Derechos Humanos sobre la aplicación del plan de acción para el decenio de las Naciones Unidas para la Educación en la esfera de los Derechos Humanos. Accesible en: http://www.un.org/spanish/aoutun/hrigts.htm. Fecha de consulta: 8-3-2009.

NACIONES UNIDAS (2006), Plan de acción: Programa mundial para la educación en derechos humanos: Primera etapa, Nueva York, Naciones Unidas. Accesible en: http://www2.ohchr. org/spanish/issues/education/training/planaction.htm. Fecha de consulta: 8-3-2009. 